\title{
LA BILHARZIOSE VÉSICALE DANS LE SUD MAROCAIN
}

\section{(SCHISTOSOMA HAEMATOBIUM)}

\author{
Par J. CARROSSE
}

Tout comme en Tunisie, le foyer bilharzien de Marrakech fut dépisté, tout d'abord, par le réactif européen militaire (Job, 1915). Conformément aux prévisions du Prof. Brumpt (1922), lors de son passage dans le sud marocain en avril 1922, le développement progressif de l'assistance médicale indigène le révéla peu à peu à l'attention générale. Malgré la notion nouvelle du mollusque intermédiaire nécessaire à l'évolution de Schistosoma hæmatobium, la délimitation du foyer fut assez lente et le plan de recherches de Brumpt, Grall et Gouzien ne put être réalisé immédiatement.

En décembre 1929, je relevai onze cas d'hématurie bilharzienne chez de jeunes soldats européens, contaminés au cours de l'été 1928 dans les citernes du camp du Gueliz. J'étendis alors mon enquête de «mireur d'urines » au bataillon de Sénégalais de la garnison. Dans le même but, Barnéoud (1929) la poursuivit en milieu civil, s'attachant surtout à certains groupements indigènes et aux douars suburbains de la grande agglomération musulmane (140.000 habitants). Il releva 210 eas en 9 mois avec les pourcentages suivants :

Cireurs et commissionnaires (Place Djema-el-Fna) $\ldots \ldots \ldots \quad 37,93 \quad 0 / 0$

Laveuses, ouvrières agricoles ................... $4,030 / 0$

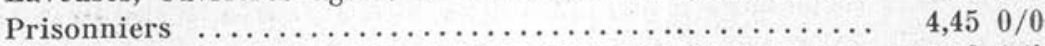

Miséreux de la station d'épouillage (Charrige el Begar) . $\quad 17,73$ 0/0

Dans le même temps, je réunis 30 observations militaires :

Cas exogènes, $6^{\circ}$ sénégalais $\ldots \ldots \ldots \ldots \ldots \ldots \ldots \ldots$ 15 (pas de $S$. mansoni).

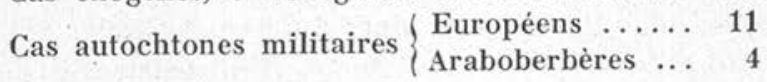

Il ne fut pas possible de déterminer le rôle exact des premiers dans la dissémination des œufs de Schistosoma. Je crois à une importation soudanaise beaucoup plus ancienne. Cette hypothèse sera vérifiée si la bilharziose vésicale est retrouvée dans le Sahara marocain (Drâa, Todra, Tafilalet), sur les pistes qui amenèrent du

Annales de Parasitologie, T. VIII, N ${ }^{\circ} 2 .-1^{\mathrm{er}} \operatorname{mars} 1930$, p. 161-164. 11. 
Soudan les Almoravides (1065) ou mème les soldats et les captifs du sultan Ahmed el Mansour après la conquête de Tombouctou (1591). En ce cas, le chaînon marocain relierait assez bien les foyers hispano-portugais de la bilharziose aux autres foyers de l'Afrique du Nord (Egypte ou Soudan).

A Marrakech, comme en Tunisie, le mollusque intermédiaire vecteur est encore Bullinus contortus (Michaud), infesté de furcocercaires dans la proportion de 5 à $60 / 0$ (Marais de Bab Robb, mai 1929).

Bien que ses gîtes soient assez nombreux et abondants dans l'Atlas, le Planorbis metidjensis (Forbes), hôte intermédiaire au Portugal, ne semble pas devoir être mis en cause. A Telouelt (Kasbah Glaoua) et dans l'Ounein (pays goundafa), je n'ai pas trouvé de bilharziens et les Planorbis ne sont pas infestés. Noirs et métis y sont fort nombreux. Il est très possible cependant que l'adaptation du trématode parasite à Planorbis metidjensis se soit réalisée sur le sol marocain et la répartition de ce mollusque est intéressante à préciser, en vue de l'adaptation éventuelle de Schistosoma mansoni, inconnu au Maroc jusqu'ici, en dehors des cas exogènes observés parfois chez les tirailleurs sénégalais.

Sur notre chiffre de 210 bilharziens, 8 résultats concernent des gens du Souss (pays au sud de l'Atlas), et j'ai donné, avec Barnéoud, les raisons nous permettant de croire qu'un certain nombre d'entre eux étaient contaminés dans leur pays d'origine et non pas à Marrakech.

Il est assez malaisé en général d'établir le curriculum vitæ ou les antécédents morbides d'un indigène nomade, surtout lorsqu'il s'agit d'une affection vésicale d'allure torpide, fort bien tolérée dans ses débuts.

Je crois cependant que l'anamnèse du cas suivant établit assez bien l'existence d'un foyer local de cette affection dans la région de Tiznit. Cette manière de procéder par renseignements, de provenance parfois lointaine, a été utilisée par les médecins tunisiens et les élèves de Ch. Nicolle. J'y ai eu recours avec L. Ferron pour démontrer l'existence d'un foyer dans la zone Ouezzan-Souk el Arba du Gharb (1929), mais il s'agissait alors d'un sous-officier aviateur, et ses déclarations furent très exactement vérifiées. Il n'en est pas de même du cas suivant, qui garde cependant toute sa valeur.

Observation. - Ahmed ben Lahoussine, âge présumé 26 ans, fils de Lahoussine ben Hadj Hamou, douar Boujaïla, fraction des Izgan, tribu des Erzmouka (entre Taroudant et Tiznit).

Notre malade a deux mois de service au $2^{\mathrm{e}}$ tirailleurs marocains 
(Marrakech), et c'est là son premier engagement. Il est à noter d'ailleurs qu'il a été hospitalisé, dès la visite d'incorporation, pour abcès froid et fistule axillaire; son engagement va ètre annulé par réforme définitive. Il offre, par ailleurs, une hématurie terminale intense. Nombreux œufs de Schistosoma hæmatobium. Eosinophilie : 25 0/0. Rien dans les selles. Pas de bacilles de Koch dans les urines. Il reçoit une série d'injections intraveineuses d'émétique (Stibyal) aux doses croissantes de 0 gr. 03, $0,06,0,09,0,12 \ldots$, etc., jusqu'à la dose totale de 1,20 et il part guéri de son hématurie.

Notre homme est un Ksourien berbère, d'esprit vif et délié, parlant et comprenant bien l'arabe. Depuis que sa tribu est soumise, elle a été rattachée au Bureau des Renseignements de Tiznit (caïd Si Mohamed Berbouchi dit Berabich). C'est un sédentaire qui n'a pour ainsi dire pas quitté le village paternel jusqu'à l'âge de 22 ans. Il se baignait dans les mares ou les canaux d'irrigation (seguias) de l'Oued Ouldghras. Il a quitté son village pour se rendre à Mogador (Souira), et ce fut son premier voyage. Il y passa 40 jours seulement et ne s'est baigné qu'à la mer. Il rentra dans sa tribu comme élève thaleb à la Zaouia de Tisguin (près d'Erzmouka), et y est resté jusqu'à son engagement. C'est là que l'hématurie a débuté, il y a deux ou trois ans, soit cinq mois après son retour de Mogador. Il confirme que, depuis son voyage, il n'est pas revenu au nord de l'Atlas, ni à Mogador, ni ailleurs.

Le début de l'affection (2 ans), le très court séjour actuel à Marrakech ( 2 mois) mettent hors de cause cette ville ou sa région. L'incubation de la bilharziose vésicale réclame 4 à 5 mois. A Mogador, le gîte à bullins de l'embouchure de l'Oued-Ksob est très connu. Entre Mogador et Agadir ; à Tamanar (Dar-caid-Guellouli) et à Tassila N. Teguedja, le bullin est plus abondant encore dans les ghedirs perennes de la région (1): Bullinus contortus Michaud et Bullinus dybovskyi Fischer.

Le $\mathrm{D}^{r}$ Bouveret, que les indigènes viennent consulter de fort loin, n'a pas eu l'occasion de signaler dans sa région l'hématurie bilharzienne. Moi-même n'ai pas trouvé un seul pisseur de sang parmi les jeunes indigènes du village de Djabet, non plus qu'à Ain-Tafetecht. Je n'ai pu visiter les points d'eau d'Harrazin, Ain-el-Hadjar et Taïtoust que me signala Bouveret et n'ai examiné que 27 échantillons d'urines : soit 20 à Ain-Tafetecht, où le bullin est rare et 7 seulement à Djabet. J'aurais dû arriver à la centaine.

En somme, mon cas relève plutòt du Souss, dans la zone Taroudant-Tiznit. Des questions épidémiologiques d'ordre beaucoup plus urgent retiennent, il est vrai, l'attention de nos confrères dans

(1) Echantillons du C $\mathrm{C}^{\mathrm{t}}$ Deschazeaux, Inspecteur des Eaux-et-Forêts de la région de Marrakech. 
une région à peine soumise. La bilharziose vésicale n'est certes pas un problème de tout premier plan, mais il ne semble pas devoir être négligé. Grall, Brumpt, Gouzien l'avaient prévu en proposant une enquête démographique très poussée au Maroc comme en A.-O.-F. L'observation de Dimitracof (1924) le confirme également.

C'est une maladie à surprises. Notre observation appelle de nouvelles recherches. La carte de la bilharziose vésicale marocaine est à dresser.

\section{RÉSU MÉ}

Ce travail donne une revue des connaissances actuelles sur Ia distribution géographique de la bilharziose vésicale dans le sudmarocain. Il est accompagné d'une observation.

\section{BiBLIOGRAPHIE}

Brumpt (E.). - La bilharziose au Maroc. Répartition du Bullinus contorlus et du Planorbis métidjensis. Etude épidémiologique comparée du foyer tunisien de Gafsa et du foyer marocain de Marrakech. Bull. Soc. Path. Exot.. XV, 1922 , p. $632-661$.

Carrosse et Barnéoud. - Enquête sur la bilharziose vésicale à Marrakech. Arch. Inst. Pasteur Algérie, VII, p. 51-78, 8 pl., 1929.

Dimıtracof. - Un cas de bilharziose vésicale européenne. Bull. Soc. Méd. Hôp. Paris, XXIII, 1924, p. 158.

Ferron (L.) et Carrosse. - Un foyer de bilharziose vésicale à Ouezzan. Maroc médical, juillet 1929 .

Јов (E.). - Note sur la bilharziose au Maroc. Bull. Mém. Soc. Méd. Hôp. Paris. XXXI, 1915, p. 1282-1288. 